\title{
Mitral Prosthesis Opening and Flow Dynamics in a Model of Left Ventricle: An In Vitro Study on a Monoleaflet Mechanical Valve
}

\author{
FREDERIC MOURET,,$+\ddagger$ LYES KADEM, ${ }^{*}$ ERIC BERTRAND, ${ }^{*}$ JEAN G. DUMESNIL, ${ }^{\dagger}$ \\ PHILIPPE PIBAROT, ${ }^{\dagger}$ and REGIS RIEU*,
}

\begin{abstract}
A complete understanding of the flow past a mitral valve prosthesis require a new generation of pulse duplicators and more realistic flow conditions. The objective of this study is to describe the opening kinetics of a monoleaflet Medtronic Hall 27-mm mechanical valve in mitral position and to determine the flow pattern within the left ventricle using particle image velocimetry (PIV) for different instants during the cardiac cycle. At the onset of diastolic phase, the flow goes through the major orifice and then through the minor orifice. The two jets generated induce two counterclockwise vortices within the ventricle, which can lead to a partial closure of the valve during mitral $\mathrm{E}$ wave deceleration. During diastasis and mitral A wave, only one vortex persists in the ventricle and pushes the valve disk backward at the end of the diastolic phase. The valve disc never reached its maximum opening $\left(65^{\circ} \mathrm{vs} .75^{\circ}\right)$. This study underscores that the flow past a monoleaflet valve in mitral position is highly dependent on the complex interaction between the inflow, the vortices development within the left ventricle, and the gravity. Such a complex interaction can only be highlighted using new generation of pulse duplicators.
\end{abstract}

Key words: cardiovascular simulator; mitral valve prostheses; particle image velocimetry; valve implantation.

\footnotetext{
* Cardiovascular Biomechanics Laboratory, IRPHE - CNRS UMR 6594, Université de la Méditerranée, Marseilles, France.

${ }^{\dagger}$ Quebec Heart Institute, Laval Hospital, Laval University, Sainte-Foy, Quebec, Canada.

${ }^{\ddagger}$ PROTOMED, Marseilles, France.

${ }^{\S}$ To whom correspondence should be addressed at École Généraliste d'Ingénieurs de Marseille, Equipe de Biomécanique Cardio-vasculaire IRPHE, IMT - Technopôle de Château Gombert, 13383 Marseille cedex 13 - France. E-mail: rieu@mecabio.com
}

\section{INTRODUCTION}

Heart valve prostheses are used to replace native mitral valves when they cannot be repaired. The choice of the type of the prosthesis (mechanical or tissue) is left to the surgeon appreciation depending on the patient (Senthilnathan et al., 1999). Several studies have focused on the flow across the mitral valve prosthesis to determine the impact of its orientation with respect to the rotational axis, or to estimate the valve prosthesis degree of obstruction (Björk et al., 1973; Chandran et al., 1991; Garitey et al., 1995; Haggag, 1990; Pop et al., 1989; Rashtian et al., 1986; Van Rijk-Zwikker et al., 1996). However, most of these studies were performed under nonphysiological flow conditions. Nowadays, standard requirements for in vitro testing of artificial valve tend toward simulation of more realistic flows. The reproduction of physiological flow through the valves requires the use of new generation simulators that activate both the ventricle and the atrium, and thus provide realistic mitral flow with $E$ and $A$ waves separated by a diastasis (Cassot et al., 1985; Mouret et al., 2000; Naemura et al., 1997; Verdonck et al., 1992). The objective of this study was to describe the valve opening kinetics of a monoleaflet mechanical valve inserted in the mitral position in a left heart pulse duplicator as well as to determine its impact on the flow distribution pattern in the left ventricle using particle image velocimetry (PIV).

\section{METHODS}

The left heart pulse duplicator used for this study has been previously described and validated (Mouret $e t$ al., 
2000). Since this duplicator has an atrioventricular dual activation system, both atrial and ventricular contractions can be controlled independently, which ensures the reproduction of physiological inflow signals through the left ventricular cavity. The dual activation simulator mainly consists of a ventricular activation device, an atrial activation device, a systemic circulation model, and a computerized driving interface. In order to simulate ventricular dynamics, a pump activates the "extracirculatory" fluid (water + glycerol) from an open tank to a box containing the ventricle model. When the fluid is injected in the ventricular box during the systolic period, it compresses the ventricle and the "intracirculatory" fluid contained in the ventricle is ejected into the aorta. To the opposite in the diastolic period, the extracirculatory fluid is extracted from the box, the ventricle expands, and the intracirculatory fluid contained in the left atrium is sucked into the ventricle. This simulates the passive ventricular filling. The same principle is used to activate the atrium model and thus simulate the atrial contraction resulting in an active ventricular filling. The atrial and ventricular anatomically shaped moldings are made from successive soakings in Silopren (General Electric Bayer Silicones). The models of the cavities are therefore compliant and transparent. The fluid used for the tests is a $40 \%$ glycerol/water solution reproducing blood viscosity $(4 \mathrm{cP})$.

Particle Image Velocimetry (PIV) (TSI Inc., France) is an optical method to measure instantaneous fluid velocity in a whole plane (Funes-Gallanzi, 1998). The flow is highlighted with a pulsed laser light. The seeding particles contained in the fluid, reflect laser light toward a digital camera. Two successive images are recorded, sampled, and cross-correlated. It is therefore possible to know the displacement of particles from one frame to the other and to compute the velocity vectors. Only one plane in the ventricle has been studied (see Fig. 1). This plane cuts the mitral and aortic annulus planes and passes through the apex. To eliminate any potential optical distortion, we used the same fluid in (intracirculatory) and outside (extracirculatory) the ventricle. The seeding particles were Amberlite ${ }^{\circledR}$ Resin, neutrally buoyant, with a mean diameter of $50 \mu \mathrm{m}$. Velocity maps were generated

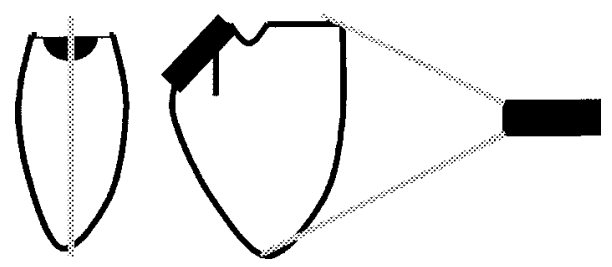

Figure 1. Scheme of the position of the measurement plane in the ventricle. with an interrogation size of 64 pixel $\times 64$ pixel, with a $50 \%$ overlap, thereby providing a spatial resolution of $2.8 \mathrm{~mm} \times 2.8 \mathrm{~mm}$. The velocities were averaged on 50 cardiac cycles. The time between two frame exposures was set to $600 \mu$ s as a compromise between velocities in the ventricle and the available power of the laser (Argon-ion Spectra Physics $5 \mathrm{~mJ}$ ).

The cardiac frequency was set at 70 beats per min. The mean cardiac output was $5 \mathrm{~L}$ per min. In accordance with clinical observations, the $E / A$ ratio for the simulated mitral flow was close to 2 . The cardiac cycle was divided in 17 instants separated from each other by $50 \mathrm{~ms}$.

A mechanical valve Medtronic Hall size 27 was tested. Figure 1 shows the orientation used for the valve. The greater orifice of the Medtronic Hall valve was oriented toward the posterior wall as previously recommended (Björk et al., 1973; Pop et al., 1989). The aortic valve was a Björk Shiley size 27.

To detect the position of the disc at the instant of measurement, the IMAQ Vision Builder software (National Instruments) was used. With this image analysis package, the edge of the disc was detected, for each instant, on each frame recorded during PIV measurements. The position was then converted to an angle between the disc and the mitral plane.

During experiments the transmitral flow was measured using an electromagnetic flowmeter (Carolina Medical SR670, accuracy 1\%) positioned just before the mitral valve. Pressure measurements were performed using Millar catheters MPC 500 (accuracy $0.5 \%$ ) at the level of the ascending aorta (for aorta pressure), ventricle apex (for ventricular pressure), and within the atrium model (for atrium pressure).

\section{RESULTS}

\section{Flow Pattern Downstream From the Medtronic Hall Valve}

The cardiac cycle is described below from the instant $100 \mathrm{~ms}$, which corresponds to the onset of diastole. At valve opening, a strong flow goes through the major orifice of the valve, there is almost no flow through the minor orifice (Fig. 2, instant $100 \mathrm{~ms}$ ). With the increase in flow velocity ( $E$ wave), a flow jet can be noticed through each orifice of the valve (Fig. 2, instant $150 \mathrm{~ms}$ ). Two counterclockwise vortices appear in the vicinity of the jets. The first vortex, located along the apex side, is induced by the wake of the valve and velocity discontinuity on both sides of the disk. The second vortex, located along the aortic side, is likely induced by viscosity between the flow jet 

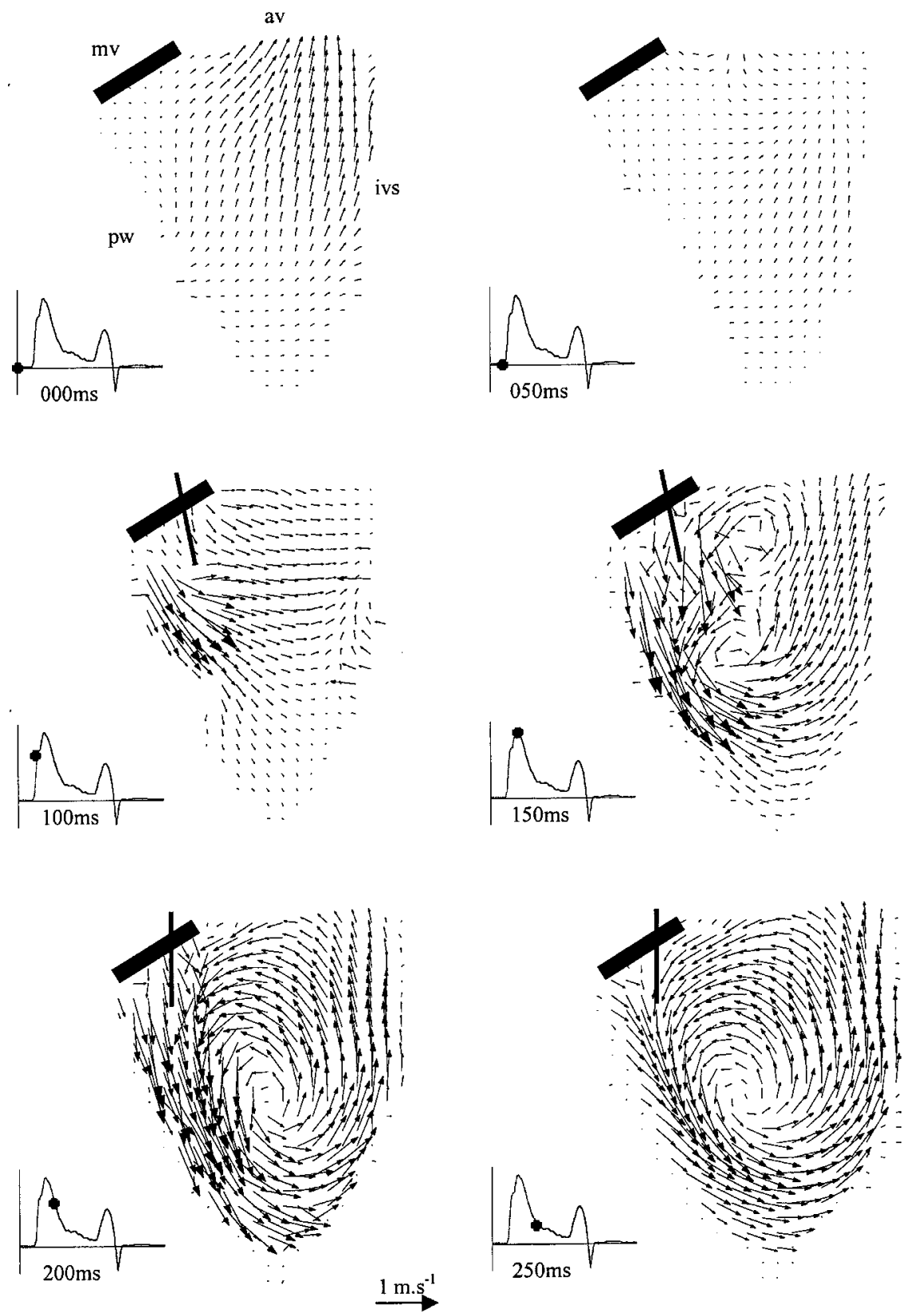

Figure 2. Velocity patterns in the ventricle from instant $000 \mathrm{~ms}$ to $250 \mathrm{~ms}$. On all frames, the mitral valve (Medtronic Hall) is represented with its disc opened or not. mv: mitral valve; av: aortic valve; ivs: interventricular septum; pw: posterior wall.

coming from the minor orifice and the stagnant fluid at the entry of left ventricular outflow tract. Because of the geometry of the ventricle and the asymmetric entry of the flow mainly through the major orifice, the former vortex alone is convected in the ventricle during diastasis (Fig. 2, instant $200 \mathrm{~ms}$ ). Moreover, mitral flow deceleration can lead to a partial closure of the valve that could favor vor- ticity emission, increasing shear stress at leaflet tip. The small vortex below the minor orifice is absorbed by the main vortex, because mitral flow deceleration leads to a decrease in flow velocity through the minor orifice. During diastasis (from Fig. 2, instant $250 \mathrm{~ms}$ to Fig. 3 instant $450 \mathrm{~ms}$ ), vortex circulates within the whole ventricle and flow velocities decrease due to mitral flow deceleration. At 

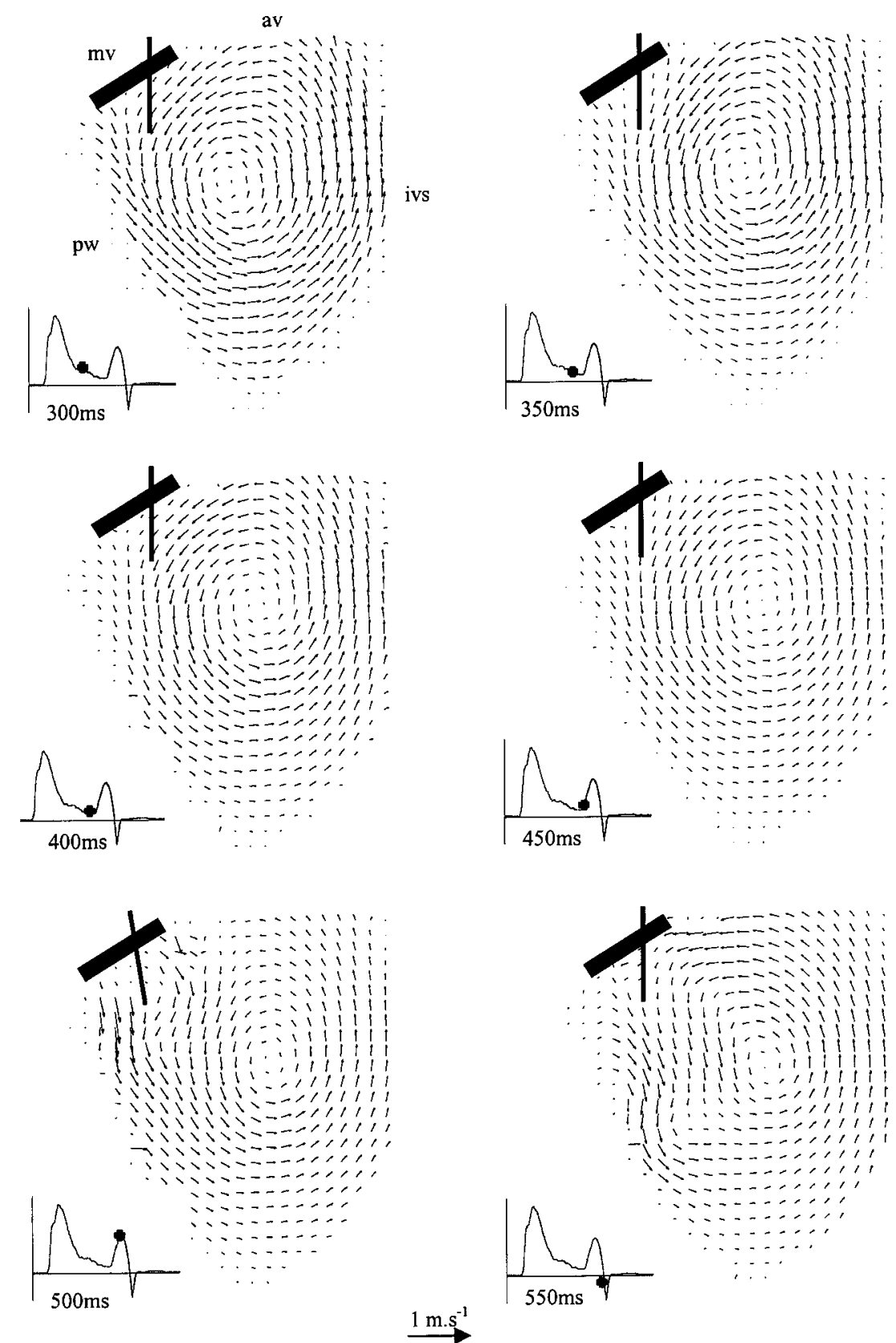

Figure 3. Velocity patterns in the ventricle from instant $300-550 \mathrm{~ms}$. On all frames, the mitral valve (Medtronic Hall) is represented with its disc opened (diastole), mv: mitral valve; av: aortic valve; ivs: interventricular septum; pw: posterior wall.

the time of atrial contraction (Fig. 3, instant $500 \mathrm{~ms}$ ) flow through both orifices feeds the main vortex. In contrast with what observed at the onset of diastole, the inertia of the main vortex prevents the occurrence of a small vortex downstream to the minor orifice and the flow velocities are oriented toward its rotational sense. At the end of the $A$ wave (Fig. 3, instant $550 \mathrm{~ms}$ ), fluid begins to push the disk to its closed position. The inward displacement of the ventricular walls directs remaining mitral flow. At the onset of systolic ejection, main flow establishes along the interventricular septum (Fig. 4, instant $600 \mathrm{~ms}$ ). Throughout the systole (Fig. 4, from instant $650 \mathrm{~ms}$ to instant $800 \mathrm{~ms}$ ), vortex core moves under mitral valve where it vanishes in telesystole. This movement of the core may be due to 

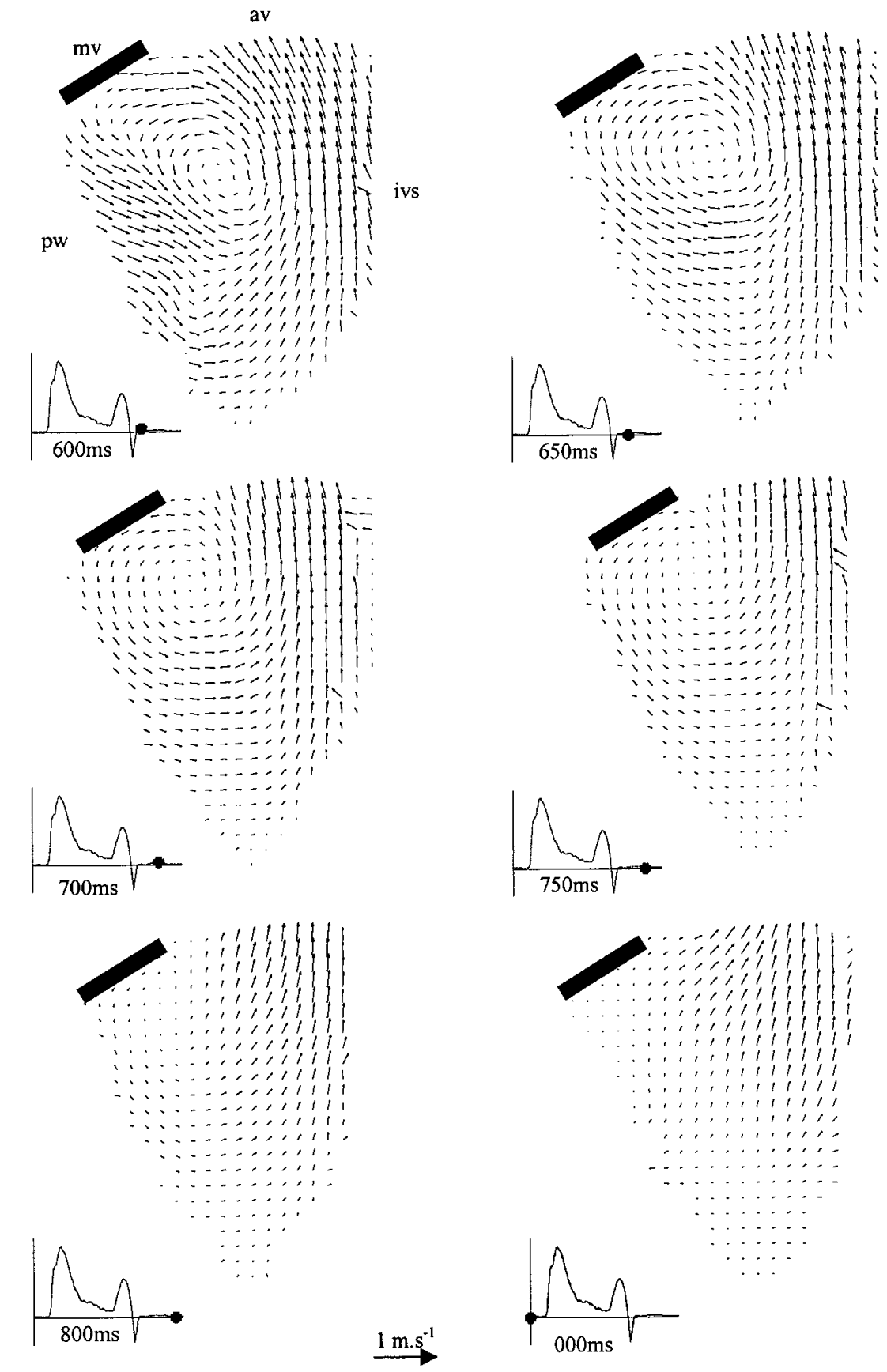

Figure 4. Velocity patterns in the ventricle from instant $600 \mathrm{~ms}$ to $000 \mathrm{~ms}$. On all frames, the mitral valve (Medtronic Hall) is represented with its disc closed (systole), mv: mitral valve; av: aortic valve; ivs: interventricular septum; pw: posterior wall.

the combined effect of posterior wall deformation and to the orientation of the ejection flow in the aorta. During isovolumic relaxation (Fig. 2, instant $050 \mathrm{~ms}$ ), velocities in the ventricle remain low. A mild regurgitation can be observed at the aortic valve.

\section{Position of the Disc of the Medtronic Hall Valve}

When analyzing PIV pictures, it is possible to detect the angle of the leaflet with respect to the mitral annulus plane. Figure 6 superposes the angle of the leaflet in the 
a)

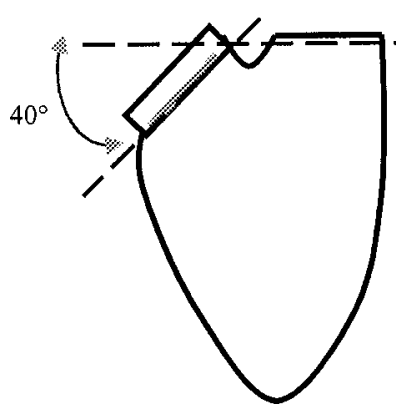

b)

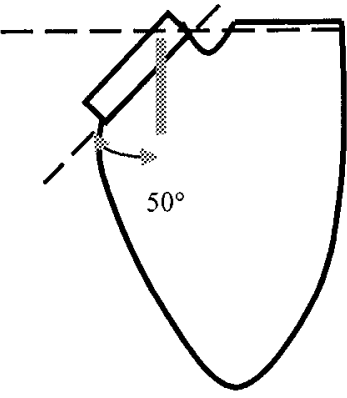

c)

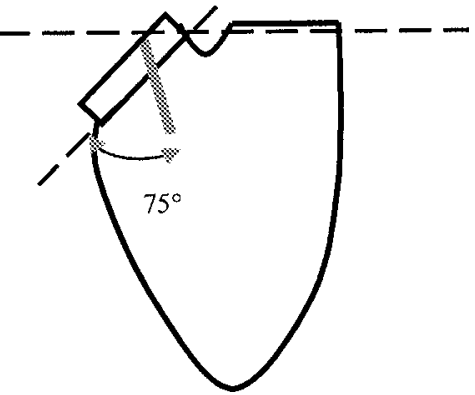

Figure 5. Disc angle and its standard deviation on 50 frames and corresponding mitral flow (black line).

time in comparison with the mitral flow recorded with the electromagnetic flowmeter.

The Medtronic valve is set on the Dual Activation Simulator (DAS) with an angle of $40^{\circ}$ with respect to the horizontal plane. A disc opening angle of $50^{\circ}$ therefore corresponds to the vertical position where the disc is submitted only to gravity (Fig. 5). First, the angle of the leaflet in our measurements never reaches the maximum $\left(75^{\circ}\right.$ for the Medtronic Hall valve). From the instant $100-150 \mathrm{~ms}$, the angle decreases whereas the flow is still accelerating; this is probably due to the fact that the vortices created at the tip of the leaflet and behind the small orifice push the leaflet backward. With flow deceleration, an equilibrium seems to be reached at the instant $200 \mathrm{~ms}$, the disc is almost vertical with a very low standard deviation in its angle. That is to say that the forces due to the incoming flow are compensated by gravity and the vortex behind the disc. During diastasis (instants $250-450 \mathrm{~ms}$ ) the disc oscillates around the vertical position with a nonnegligible standard deviation. During this period, the disc is not anymore directed by the incoming flow, which is very low, but by the gravity and the rotating flow in the ventricle. The large standard deviation may be related to the passive behavior of the leaflet during diastasis. At atrial contraction (instant 500), the reentrant flow in the cavity, which pushes the leaflet toward its open position, is associated with an increase in the angle and a lower

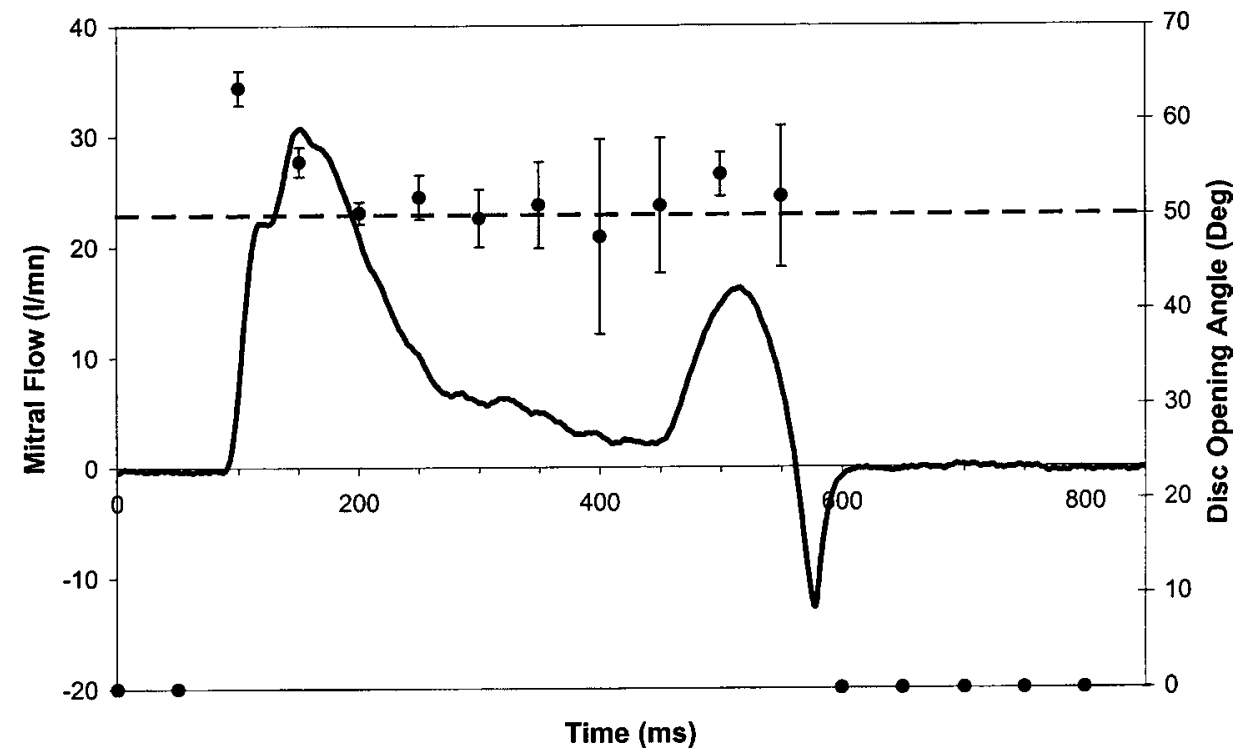

Figure 6. Angles description in various positions of the leaflet, (a) $0^{\circ}$ disc in closed position, (b) $50^{\circ}$ disc in vertical position, (c) $75^{\circ}$ disc in maximal opening position. 
standard deviation. As for the beginning of diastole, the timing of peak wave velocity recorded by the flowmeter does not correspond to the time when the valve is fully opened. During flow deceleration, at valve closure, the large standard deviation observed indicates that the position of the disc is very difficult to predict. It can be explained by the fact that the closing velocity of the leaflet is very high and not reproducible one cycle after the other.

\section{DISCUSSION}

The major contribution of this study was to describe the ventricular flow pattern downstream from a monoleaflet mechanical valve in a left heart model, which accurately reproduces the physiological inflow and outflow conditions of the normal human heart.

\section{Opening Dynamics of Monoleaflet Mechanical Valves}

In these measurements, the valve appears not to open completely. This is certainly due to the fact that the instants of measurements do not correspond to the time when the valve is maximally opened. Indeed, the opening time for a valve is moreover around $25-35 \mathrm{~ms}$, which is lower than the time between two acquisitions $(50 \mathrm{~ms})$. We can see from this analysis that the gravity plays an important role in tilting disc position during diastasis. This could be all the more important when considering bileaflet valves where one of the leaflets could close during diastasis favoring thrombus formation. Further studies could be planned to study the effect of gravity on the behavior of the valves. This study reinforces the need to simulate a physiological flow through the mitral valve when qualifying heart valve prostheses.

\section{Comparison With Previous Clinical Studies}

Two techniques can be used to study the flow velocity distribution within the left ventricle: Dopplerechocardiography and Magnetic Resonance Imaging (MRI). The first one seems to be less precise than the second but is much more used clinically due to its lower cost. Rodevand et al. (1999) described the left ventricular flow pattern within the ventricle in healthy subjects with color Doppler. During acceleration of early diastolic filling flow, velocities are directed toward the apex. At the peak of $E$ wave, a decreasing velocity gradient is observed between mitral valve and the apex. When the flow decelerates, the anterior leaflet of the mitral valve begins to move toward the closed position, backward flow velocities appear in the left ventricular outflow tract, and a vortex appears at the leaflet tip. Another vortex is also visible under the posterior leaflet. During diastasis, filling velocities are very low as the one located along the septal wall. This suggests the presence of a large vortex in the whole ventricle. In some patients, a contrarotative circulation is observed at the level of the apex. At the beginning of the atrial contraction, some substantial velocities are consistently recorded through the mitral orifice, creating during $A$ wave and its deceleration the same flow pattern as in early diastole. Kim et al. (1995) have performed MRI measurements in young and healthy subjects. They recorded a mild regurgitation through the mitral valve at the time of valve closure. The systolic ejection is characterized by a flow converging toward the left ventricular outflow tract from the whole ventricle. After peak systole, the flow decreases until aortic valve closure without any significant regurgitation. A small eddy occurs below the valve at this moment. No significant flow is observed during isovolumic relaxation. At valve opening, a large flow goes through the mitral valve toward the apex. During $E$ wave deceleration, a vortex occurs at leaflet tip and directs flow toward the posterior wall. A weak recirculation can be observed under the posterior leaflet. During diastasis, no flow is noticed through the mitral valve. Atrial contraction is associated with the onset of a new incoming flow into the ventricle before valve closure. The findings of Kilner et al. (2000) with MRI are also in agreement with Kim et al. observations. In our in vitro experiments, the vortices seem to occur before the deceleration of the $E$ wave which is somewhat earlier than what is reported in vivo. This is probably related to higher vorticity at the leaflet tips. Indeed as the valve is not fully opened at the moment of $E$ wave, vorticity is increased by the angle of the disc and it results in higher shear stress. Nonetheless, the in vivo data reported in these studies are very consistent with the data obtained with our dual activating left heart simulator thus confirming the clinical relevance of these in vitro data.

\section{CONCLUSION}

The Dual Activation Simulator used in this study reproduces the left heart circulation of the human being in the upright position. The major findings of this study are: The monoleaflet valve implanted in the optimal position 
and tested under normal flow conditions (i.e. similar to the normal resting flow conditions across human mitral valve) does not appear to reach the maximum possible opening, i.e. a disc opening angle of $75^{\circ}$. The maximal opening angle observed in this study was around $65^{\circ}$. Due to the limited frame rate of the PIV system, it is possible that we have not detected the true maximal opening angle in early diastole. One can thus not exclude that the maximal opening angle was higher than $65^{\circ}$ and possibly reached the maximum possible angle of $75^{\circ}$ during the early acceleration phase of the $E$ wave. Nonetheless, these experiments also show that during most of diastolic period, the opening angle remains lower than $60^{\circ}$. This finding indicates that, at normal resting flow conditions, the Medtronic Hall 27 valve has some substantial opening reserve. This suggests that this valve has potential to accommodate higher cardiac output such as may occur during exercise. The results of this study also suggest that the maximal opening of the disc and the peak transvalvular velocity does not occur at the same time. In fact, the disc reaches its maximal opening before the occurrence of the peak velocity. This may be due to the fact, that the discs of mechanical valves, as opposed to those of bioprosthetic valves, have a low opening inertia. The valve disc opens rapidly at the onset of $E$ wave and it reaches its maximal opening angle $\left(65-75^{\circ}\right)$ generally within $25-$ $35 \mathrm{~ms}$. When the flow rate increases across the valve orifice during $E$ wave acceleration phase, vortex develop downstream from the valve, thus pushing back the disk toward a lower opening angle $\left(50-55^{\circ}\right)$. The valve orifice therefore becomes smaller whereas the flow is still accelerating. This may explain why the peak $E$ wave (and $A$ wave) velocity occurs approximately $30 \mathrm{~ms}$ after the maximal opening of the disc. This study shows that in upright position, the position of the disc of monoleaflet mechanical valves remains widely open during diastasis, likely due to the effect of gravity on the disc. In contrast, previous studies have reported that at least one of the two hemidiscs of bileaflet mechanical valves close during diastasis, which could, theoretically predispose to thrombus formation. Further studies are needed to determine if this difference between monoleaflet and bileaflet mechanical valves in regard to the opening dynamics during diastasis may translate into significant difference in regard to the occurrence of thromboembolic events in vivo. As emphasized by the results of this study, gravity seems to have an important impact on the opening dynamics of monoleaflet mechanical valves. This contrasts with bioprosthetic valves or native valves where the effect of gravity is minimal

\section{REFERENCES}

Bellhouse BJ. Fluid mechanics of a model mitral valve and left ventricle. Cardiovasc Res 6: 199-210, 1972.

Björk VO, Book K, and Holmgren A. Significance of position and opening angle of the Björk-Shiley tilting disk valve in mitral surgery. Scand J Thorac Cardiovasc Surg 7: 187-201, 1973.

Cassot F, Morvan D, Issartier P, and Pelissier R. New versatile physical model fitting the systemic circulation accurately. Med Biol Eng Comput 23: 511-516, 1985.

Chandran KB, Schoephoester R, and Dellsperger KC. Effect of prosthetic mitral valve geometry and orientation flow dynamics in a model of human left ventricle. $J$ Biomech 1: 51-65, 1991.

Funes-Gallanzi M. High accuracy measurement of unsteady flows using digital particle image velocimetry. Opt Laser Technol 30: 349359, 1998.

Garitey V, Gandelheid T, Fuseri J, Pelissier R, and Rieu R. Ventricular flow dynamics past bileaflet prosthetic heart valves. Int J Artif Organs 18: 380-391, 1995.

Haggag YA. The central axis prosthetic cardiac valve: An in vitro study of pressure drop assessment under steady-state flow conditions. J Biomed Eng 12(1): 63-68, 1990.

Kilner PJ, Yang GZ, Wilkes AJ, Mohiaddin RH, Firmin DN, and Yacoub MH. Asymetric redirection of flow through the heart. Nature 404: 759-761, 2000.

Kim WY, Walker PG, Pedersen EM, Poulsen JK, Oyre S, Houlind K, and Yoganathan AP. Left ventricular blood flow patterns in normal subjects: A quantitative analysis by three dimensional magnetic resonance velocity mapping. $J$ Am Coll Cardiol 26: 224-238, 1995.

Mouret F, Garitey V, Gandelheid T, Fuseri R, and Rieu R. A new dual activation simulator of the left heart that reproduces physiological and pathological conditions. Med Biol Eng Comput 38: 558-561, 2000.

Naemura K, Sonderegger M, Umezu M, et al. Study of effect of atrial contraction in mitral prosthetic valve by high speed video camera. Artif Organs 21: 300-305, 1997.

Pop G, Sutherland GR, Roedland J, Vletter W, and Bos E. What is the ideal orientation of a mitral disc prosthesis. An in vivo haemodynamic study based on colour flow imaging and continuous wave Doppler. Eur Heart J 10: 346-353, 1989.

Rashtian MY, Stevenson DM, Allen DT, et al. Flow characteristics of four commonly used mechanical heart valves. Am J Cardiol 58(9): 743-752, 1986

Rodevand O, Bjornerheim R, Edvardsen T, Smiseth OA, and Ihlen H. Diastolic flow pattern in the normal left ventricle. $J$ Am Soc Echocardiogr 12: 500-507, 1999.

Senthilnathan V, Treasure T, Grunkemeier G, and Starr A. Heart valves: Which is the best choice? Cardiovasc Surg 7: 393-397, 1999.

Van Rijk-Zwikker GL, Delemarre BJ, and Huysmans HA. The orientation of the bi-leaflet Carbomedics valve in the mitral position determines left ventricular spatial flow patterns. Eur J Cardiothorac Surg 10: 513-520, 1996.

Verdonck P, Kleven A, Verhoeven R, Angelsen B, and Vandenbogaerde J. Computer-controlled in vitro model of the human left heart. Med Biol Eng Comput 30: 656-659, 1992. 